\title{
Neutrinoless double beta decay in left-right symmetric models with a universal seesaw mechanism
}

\author{
Frank F. Deppisch, ${ }^{1, *}$ Chandan Hati, ${ }^{2,3, \dagger}$ Sudhanwa Patra, ${ }^{4,6, \$}$ Prativa Pritimita, ${ }^{4, \S}$ and Utpal Sarkar ${ }^{5, \|}$ \\ ${ }^{1}$ Department of Physics and Astronomy, University College London, London WC1E 6BT, United Kingdom \\ ${ }^{2}$ Physical Research Laboratory, Navrangpura, Ahmedabad 380009, India \\ ${ }^{3}$ Indian Institute of Technology Gandhinagar, Chandkheda, Ahmedabad 382424, India \\ ${ }^{4}$ Center of Excellence in Theoretical and Mathematical Sciences, \\ Siksha 'O' Anusandhan University, Bhubaneswar 751030, India \\ ${ }^{5}$ Department of Physics, Indian Institute of Technology Kharagpur, Kharagpur 721302, India \\ ${ }^{6}$ Indian Institute of Technology Bhilai, GEC Campus, Sejbahar, Raipur, Chhattisgarh 492015, India
}

(Received 18 January 2017; revised manuscript received 22 December 2017; published 6 February 2018)

\begin{abstract}
We discuss a class of left-right symmetric theories with a universal seesaw mechanism for fermion masses and mixing and the implications for neutrinoless double beta $(0 \nu \beta \beta)$ decay where neutrino masses are governed by natural type-II seesaw dominance. The scalar sector consists of left- and right-handed Higgs doublets and triplets, while the conventional Higgs bidoublet is absent in this scenario. We use the Higgs doublets to implement the left-right and the electroweak symmetry breaking. On the other hand, the Higgs triplets with induced vacuum expectation values can give Majorana masses to light and heavy neutrinos and mediate $0 \nu \beta \beta$ decay. In the absence of the Dirac mass terms for the neutrinos, this framework can naturally realize type-II seesaw dominance even if the right-handed neutrinos have masses of a few $\mathrm{TeV}$. We study the implications of this framework in the context of $0 \nu \beta \beta$ decay.
\end{abstract}

DOI: 10.1103/PhysRevD.97.035005

\section{INTRODUCTION}

The neutrino oscillation experiments have established that the neutrinos have nonzero masses. However, the question regarding the fundamental nature of the neutrinos; whether they are Dirac [1] or Majorana [2], is yet to be answered. From a theoretical point of view several frameworks predict Majorana neutrinos, while the experimental searches are still inconclusive in this regard. To this end the detection of neutrinoless double beta $(0 \nu \beta \beta)$ decay, which requires neutrinos to be Majorana particles regardless of the underlying mechanism, plays the crucial role in confirming the nature of the neutrinos. This rare process, the conversion of two neutrons into two protons, two electrons and nothing else, if observed, would pave a path towards the search for new physics beyond the standard model (SM). Currently, the KamLAND-Zen experiment [3] (using

\footnotetext{
*f.deppisch@ucl.ac.uk

†chandan@prl.res.in

*sudha.astro@gmail.com

${ }^{\S}$ pratibha.pritimita@gmail.com

utpal@phy.iitkgp.ernet.in
}

Published by the American Physical Society under the terms of the Creative Commons Attribution 4.0 International license. Further distribution of this work must maintain attribution to the author(s) and the published article's title, journal citation, and DOI. Funded by SCOAP ${ }^{3}$.
${ }^{136} \mathrm{Xe}$ ) claims the best limit on the half-life $T_{1 / 2}$ to be less than $1.07 \times 10^{26} \mathrm{yr}$ corresponding to an upper bound on effective Majorana mass $m_{\text {eff }} \lesssim 0.06-0.17 \mathrm{eV}$, depending on the nuclear matrix element calculation used.

Models naturally accommodating neutrino masses are the need of the time and the left-right symmetric model (LRSM) [4-9] is one of the most popular candidates for this purpose. The right-handed neutrinos and mirror gauge bosons present make it quintessential to explain the $V-A$ nature of the weak interactions, tiny but nonzero masses of neutrinos [10-14] and a lot more. Moreover, the spontaneous breaking of the left-right symmetry at $\mathrm{TeV}$ scale offers a plethora of possibilities in collider phenomenology. The LHC gives a lower bound on the right-handed charged gauge boson mass $M_{W_{R}}$ of $\mathcal{O}(3 \mathrm{TeV})$ [15], while the kaon $K_{L}-K_{S}$ mass difference results in a lower bound on $M_{W_{R}}$ of $2.5 \mathrm{TeV}$ [16-19]. ${ }^{1}$ Such a low scale $W_{R}$ gauge boson associated with right-handed charged currents can give rise to new contributions to $0 \nu \beta \beta$ decay and can be accessible at the LHC. The current limits on left-right symmetry and neutrinoless double beta decay using the LHC dilepton channel data can be found in Refs. [23,24].

\footnotetext{
${ }^{1}$ Lower values of $M_{W_{R}}$ in the range $1.9-2.5 \mathrm{TeV}$ are allowed within TeV scale LRSMs with spontaneous D-parity breaking [20-22].
} 
In this work we study a LRSM framework with vectorlike fermions realizing a universal seesaw mechanism for fermion masses, except for the neutrinos. The scalar sector consists of left- and right-handed Higgs doublets and triplets, while the conventional Higgs bidoublet is absent in this scenario. We use the Higgs doublets to implement the left-right and the electroweak symmetry breaking. On the other hand the Higgs triplets with induced vacuum expectation values give Majorana masses to the light and heavy neutrinos in the absence of the Dirac mass terms. Consequently, one can naturally realize a type-II seesaw dominance in this framework even if the right-handed neutrinos have masses around a few TeV. We study its implications for the $0 \nu \beta \beta$ decay in this framework.

The outline for the rest of this paper is as follows. In Sec. II we start with a brief overview of the model, followed by discussions on the generation of fermion masses via universal seesaw in Sec. III, neutrino masses via type-II seesaw dominance in Sec. IV and the gauge bosons in Sec. V. In Sec. VI we discuss the implications for $0 \nu \beta \beta$ decay. Finally, in Sec. VII we summarize our results and conclude.

\section{LEFT-RIGHT SYMMETRY WITH VECTORLIKE FERMIONS}

The gauge group for LRSMs is $S U(2)_{L} \times S U(2)_{R} \times$ $U(1)_{B-L} \times S U(3)_{C}$. The charge equation relating the electric charge to the third component of isospin in the $S U(2) L, R$ gauge groups and the difference between baryon and lepton number $B-L$ is given by

$$
Q=T_{3 L}+T_{3 R}+\frac{(B-L)}{2} .
$$

The usual fermion content of the model is

$$
\begin{gathered}
Q_{L}=\left(\begin{array}{l}
u_{L} \\
d_{L}
\end{array}\right) \equiv\left[2,1, \frac{1}{3}, 3\right], \\
Q_{R}=\left(\begin{array}{l}
u_{R} \\
d_{R}
\end{array}\right) \equiv\left[1,2, \frac{1}{3}, 3\right], \\
\ell_{L}=\left(\begin{array}{c}
\nu_{L} \\
e_{L}
\end{array}\right) \equiv[2,1,-1,1], \\
\ell_{R}=\left(\begin{array}{c}
\nu_{R} \\
e_{R}
\end{array}\right) \equiv[1,2,-1,1],
\end{gathered}
$$

where the numbers in brackets correspond to the transformations under $S U(2)_{L} \times S U(2)_{R} \times U(1)_{B-L} \times S U(3)_{C}$. We also consider additional vectorlike quarks and charged leptons [25-28],

$$
\begin{aligned}
U_{L, R} & \equiv[1,1,4 / 3,3], \\
D_{L, R} & \equiv[1,1,-2 / 3,3], \\
E_{L, R} & \equiv[1,1,-2,1] .
\end{aligned}
$$

TABLE I. Field content of the LRSM with universal seesaw.

\begin{tabular}{lcccc}
\hline \hline Field & $S U(2)_{L}$ & $S U(2)_{R}$ & $B-L$ & $S U(3)_{C}$ \\
\hline$Q_{L}$ & 2 & 1 & $1 / 3$ & 3 \\
$Q_{R}$ & 1 & 2 & $1 / 3$ & 3 \\
$\ell_{L}$ & 2 & 1 & -1 & 1 \\
$\ell_{R}$ & 1 & 2 & -1 & 1 \\
$U_{L, R}$ & 1 & 1 & $4 / 3$ & 3 \\
$D_{L, R}$ & 1 & 1 & $-2 / 3$ & 3 \\
$E_{L, R}$ & 1 & 1 & -2 & 1 \\
$H_{L}$ & 2 & 1 & -1 & 1 \\
$H_{R}$ & 1 & 2 & -1 & 1 \\
$\Delta_{L}$ & 3 & 1 & 2 & 1 \\
$\Delta_{R}$ & 1 & 3 & 2 & 1 \\
\hline \hline
\end{tabular}

In Table I, we present the field content of the left-right symmetric model with universal seesaw and their transformations under the LRSM gauge group.

We implement a scalar sector consisting of $S U(2)_{L, R}$ doublets and triplets, however the conventional scalar bidoublet is absent. We use the Higgs doublets to implement the left-right and the electroweak symmetry breaking: $H_{R} \equiv\left(h_{R}^{0}, h_{R}^{-}\right)^{T} \equiv[1,2,-1,1]$ breaks the left-right symmetry, while $H_{L} \equiv\left(h_{L}^{0}, h_{L}^{-}\right)^{T} \equiv[2,1,-1,1]$ breaks the electroweak symmetry once they acquire vacuum expectation values (VEVs),

$$
\left\langle H_{R}\right\rangle=\left(\begin{array}{c}
\frac{v_{R}}{\sqrt{2}} \\
0
\end{array}\right), \quad\left\langle H_{L}\right\rangle=\left(\begin{array}{c}
\frac{v_{L}}{\sqrt{2}} \\
0
\end{array}\right) .
$$

Note that the present framework requires only doublet Higgs fields for spontaneous symmetry breaking. However, in the absence of a Higgs bidoublet, we use the vectorlike new fermions to generate correct charged fermion masses through a universal seesaw mechanism. For the neutrinos we note that in the absence of a scalar bidoublet there is no Dirac mass term for light neutrinos and without scalar triplets no Majorana masses are generated either. To remedy this fact we introduce additional scalar triplets $\Delta_{L}$ and $\Delta_{R}$,

$$
\Delta_{L, R}=\left(\begin{array}{cc}
\delta_{L, R}^{+} / \sqrt{2} & \delta_{L, R}^{++} \\
\delta_{L, R}^{0} & -\delta_{L, R}^{+} / \sqrt{2}
\end{array}\right),
$$

which transform as $\Delta_{L} \equiv[3,1,2,1]$ and $\Delta_{R} \equiv[1,3,2,1]$, respectively. They generate Majorana masses for the light and heavy neutrinos although they are not essential in spontaneous symmetry breaking here. In the presence of the Higgs triplets, the manifestly left-right symmetric scalar potential has the form

$$
\begin{aligned}
\mathcal{L}= & \left(D_{\mu} H_{L}\right)^{\dagger} D^{\mu} H_{L}+\left(D_{\mu} H_{R}\right)^{\dagger} D^{\mu} H_{R} \\
& +\left(D_{\mu} \Delta_{L}\right)^{\dagger} D^{\mu} \Delta_{L}+\left(D_{\mu} \Delta_{R}\right)^{\dagger} D^{\mu} \Delta_{R} \\
& -\mathcal{V}\left(H_{L}, H_{R}, \Delta_{L}, \Delta_{R}\right),
\end{aligned}
$$

where the scalar potential is given by 


$$
\begin{aligned}
\mathcal{V}\left(H_{L}, H_{R}, \Delta_{L}, \Delta_{R}\right)= & -\mu^{2}\left(H_{L}^{\dagger} H_{L}+H_{R}^{\dagger} H_{R}\right)+\lambda\left[\left(H_{L}^{\dagger} H_{L}\right)^{2}+\left(H_{R}^{\dagger} H_{R}\right)^{2}\right]+\beta\left(H_{L}^{\dagger} H_{L}\right)\left(H_{R}^{\dagger} H_{R}\right) \\
& -\mu^{\prime 2}\left[\operatorname{Tr}\left(\Delta_{L}^{\dagger} \Delta_{L}\right)+\operatorname{Tr}\left(\Delta_{R}^{\dagger} \Delta_{R}\right)\right]+\lambda_{1}\left[\left(\operatorname{Tr}\left(\Delta_{L}^{\dagger} \Delta_{L}\right)\right)^{2}+\left(\operatorname{Tr}\left(\Delta_{R}^{\dagger} \Delta_{R}\right)\right)^{2}\right] \\
& +\lambda_{2}\left[\operatorname{Tr}\left(\Delta_{L}^{\dagger} \Delta_{L} \Delta_{L}^{\dagger} \Delta_{L}\right)+\operatorname{Tr}\left(\Delta_{R}^{\dagger} \Delta_{R} \Delta_{R}^{\dagger} \Delta_{R}\right)\right]+\lambda_{3}\left[\operatorname{Tr}\left(\Delta_{L}^{\dagger} \Delta_{L}^{\dagger}\right) \operatorname{Tr}\left(\Delta_{L} \Delta_{L}\right)+\operatorname{Tr}\left(\Delta_{R}^{\dagger} \Delta_{R}^{\dagger}\right) \operatorname{Tr}\left(\Delta_{R} \Delta_{R}\right)\right] \\
& +\beta_{1} \operatorname{Tr}\left(\Delta_{L}^{\dagger} \Delta_{L}\right) \operatorname{Tr}\left(\Delta_{R}^{\dagger} \Delta_{R}\right)+\beta_{2} \operatorname{Tr}\left(\Delta_{L} \Delta_{L}\right) \operatorname{Tr}\left(\Delta_{R}^{\dagger} \Delta_{R}^{\dagger}\right)+\rho_{1}\left(\operatorname{Tr}\left(\Delta_{L}^{\dagger} \Delta_{L}\right)\left(H_{R}^{\dagger} H_{R}\right)+\operatorname{Tr}\left(\Delta_{R}^{\dagger} \Delta_{R}\right)\left(H_{L}^{\dagger} H_{L}\right)\right) \\
& +\rho_{2}\left(\operatorname{Tr}\left(\Delta_{L}^{\dagger} \Delta_{L}\right)\left(H_{L}^{\dagger} H_{L}\right)+\operatorname{Tr}\left(\Delta_{R}^{\dagger} \Delta_{R}\right)\left(H_{R}^{\dagger} H_{R}\right)\right)+\rho_{3}\left(H_{L}^{\dagger} \Delta_{L} \Delta_{L}^{\dagger} H_{L}+H_{R}^{\dagger} \Delta_{R} \Delta_{R}^{\dagger} H_{R}\right) \\
& +\rho_{4}\left(H_{L}^{\dagger} \Delta_{R} \Delta_{R}^{\dagger} H_{L}+H_{R}^{\dagger} \Delta_{L} \Delta_{L}^{\dagger} H_{R}\right)+\mu\left(H_{L}^{T} i \sigma_{2} \Delta_{L} H_{L}+H_{R}^{T} i \sigma_{2} \Delta_{R} H_{R}\right)+\text { H.c. }
\end{aligned}
$$

We will assign nonzero VEVs to the Higgs doublets $H_{R}$ and $H_{L}$ and triplets $\Delta_{R}$ and $\Delta_{L}$ as follows

$$
\begin{array}{ll}
\left\langle H_{R}\right\rangle=\left(\begin{array}{c}
\frac{v_{R}}{\sqrt{2}} \\
0
\end{array}\right), & \left\langle H_{L}\right\rangle=\left(\begin{array}{c}
\frac{v_{L}}{\sqrt{2}} \\
0
\end{array}\right), \\
\left\langle\Delta_{R}\right\rangle=\left(\begin{array}{cc}
0 & 0 \\
\frac{u_{R}}{\sqrt{2}} & 0
\end{array}\right), & \left\langle\Delta_{L}\right\rangle=\left(\begin{array}{cc}
0 & 0 \\
\frac{u_{L}}{\sqrt{2}} & 0
\end{array}\right) .
\end{array}
$$

Now considering the above VEVs, the part of the scalar potential involving only triplet Higgs takes the form

$$
\begin{aligned}
\mathcal{V}\left(\left\langle\Delta_{L}^{0}\right\rangle,\left\langle\Delta_{R}^{0}\right\rangle\right)= & -\frac{1}{2} \mu^{\prime 2}\left(u_{L}^{2}+u_{R}^{2}\right)+\frac{1}{4}\left(\lambda_{1}+\lambda_{2}\right)\left(u_{L}^{4}+u_{R}^{4}\right) \\
& +\frac{1}{4} \beta_{1} u_{L}^{2} u_{R}^{2} .
\end{aligned}
$$

Next, let us parametrize $u_{L}=u \sin \alpha$ and $u_{R}=u \cos \alpha$ to obtain the extremum condition after differentiating $\mathcal{V}\left(\left\langle\Delta_{L}^{0}\right\rangle,\left\langle\Delta_{R}^{0}\right\rangle\right)$ with respect to $\alpha$ :,

$$
\left[\beta_{1}-2\left(\lambda_{1}+\lambda_{2}\right)\right] u^{4} \sin (2 \alpha) \cos (2 \alpha)=0 .
$$

The solution $\alpha=\frac{\pi}{4}$ of Eq. (10) corresponds to the minimum for the choice $\beta_{1}<2\left(\lambda_{1}+\lambda_{2}\right)$, as can be readily verified by taking a double derivative of the potential with respect to $\alpha$, whereas $\alpha=0$ and $\pi / 2$ correspond to the maximum for $\beta_{1}<2\left(\lambda_{1}+\lambda_{2}\right)$. The solution $\alpha=\frac{\pi}{4}$ of Eq. (10) implies $u_{L}=u_{R}=0$. Once we consider the full potential now to include the triplet interactions with $H_{L}$ and $H_{R}$ such that a nonzero VEV $\left\langle H_{R}^{0}\right\rangle=v_{R}$ breaks the LRSM to the SM at a high scale and $\left\langle H_{L}^{0}\right\rangle=v_{L}$ breaks the SM at the electroweak scale we can chose the induced VEVs for scalar triplets much smaller than VEVs of Higgs doublets for a range of parameters in the scalar potential, i.e $u_{L}, u_{R} \ll v_{L}, v_{R}$. Consequently, one is well justified write down the induced VEVs in the Higgs triplets as

$$
u_{L}=\frac{\mu v_{L}^{2}}{M_{\delta_{L}^{0}}^{2}}, \quad u_{R}=\frac{\mu v_{R}^{2}}{M_{\delta_{R}^{0}}^{2}},
$$

assuming that the term in the scalar potential containing the masses of the scalar triplets dominate over other competing terms.

\section{FERMION MASSES VIA UNIVERSAL SEESAW}

As discussed earlier, in this scheme normal Dirac mass terms for the SM fermions are not allowed due to the absence of a bidoublet Higgs scalar. However, in the presence of vectorlike copies of quark and charged lepton gauge isosinglets, the charged fermion mass matrices can assume a seesaw structure. The Yukawa interaction Lagrangian in this model is given by

$$
\begin{aligned}
\mathcal{L}= & -Y_{U}^{L} H_{L} \bar{Q}_{L} U_{R}+Y_{U}^{R} H_{R} \bar{Q}_{R} U_{L}+Y_{D}^{L} \tilde{H}_{L} \bar{Q}_{L} D_{R} \\
& +Y_{D}^{R} \tilde{H}_{R} \bar{Q}_{R} D_{L}+Y_{E}^{L} \tilde{H}_{L} \bar{\ell}_{L} E_{R}+Y_{E}^{R} \tilde{H}_{R} \bar{\ell}_{R} E_{L} \\
& +\frac{1}{2} f\left(\overline{\bar{\ell}_{L}^{c}} i \tau_{2} \Delta_{L} \ell_{L}+\overline{\ell_{R}^{c}} i \tau_{2} \Delta_{R} \ell_{R}\right) \\
& -M_{U} \bar{U}_{L} U_{R}-M_{D} \bar{D}_{L} D_{R}-M_{E} \bar{E}_{L} E_{R}+\text { H.c. }
\end{aligned}
$$

where we suppress the flavor and color indices on the fields and couplings. $\tilde{H}_{L, R}$ denotes $\tau_{2} H_{L, R}^{*}$, where $\tau_{2}$ is the usual second Pauli matrix. Note that there is an ambiguity regarding the breaking of parity, which can either be broken spontaneously with the left-right symmetry at around the $\mathrm{TeV}$ scale or at a much higher scale independent of the left-right symmetry breaking. In the latter case, the Yukawa couplings corresponding to the right-type and lefttype Yukawa terms can be different because of the renormalization group running below the parity breaking scale, $Y_{X}^{R} \neq Y_{X}^{L}$. Thus, while writing the Yukawa terms above we distinguish the left- and right-handed couplings explicitly with the subscripts $L$ and $R$.

After spontaneous symmetry breaking we can write the mass matrices for the charged fermions as [26]

$$
\begin{array}{rlr}
M_{u U} & =\left(\begin{array}{cc}
0 & Y_{U}^{L} v_{L} \\
Y_{U}^{R} v_{R} & M_{U}
\end{array}\right), & M_{d D}=\left(\begin{array}{cc}
0 & Y_{D}^{L} v_{L} \\
Y_{D}^{R} v_{R} & M_{D}
\end{array}\right), \\
M_{e E} & =\left(\begin{array}{cc}
0 & Y_{E}^{L} v_{L} \\
Y_{E}^{R} v_{R} & M_{E}
\end{array}\right) .
\end{array}
$$

The corresponding generation of fermion masses is diagrammatically depicted in Fig. 1. Note that we are interested in a scenario where the VEVs of the Higgs doublets are much larger than the VEVs of the Higgs triplets i.e, $u_{L} \ll v_{L}, u_{R} \ll v_{R}$. 

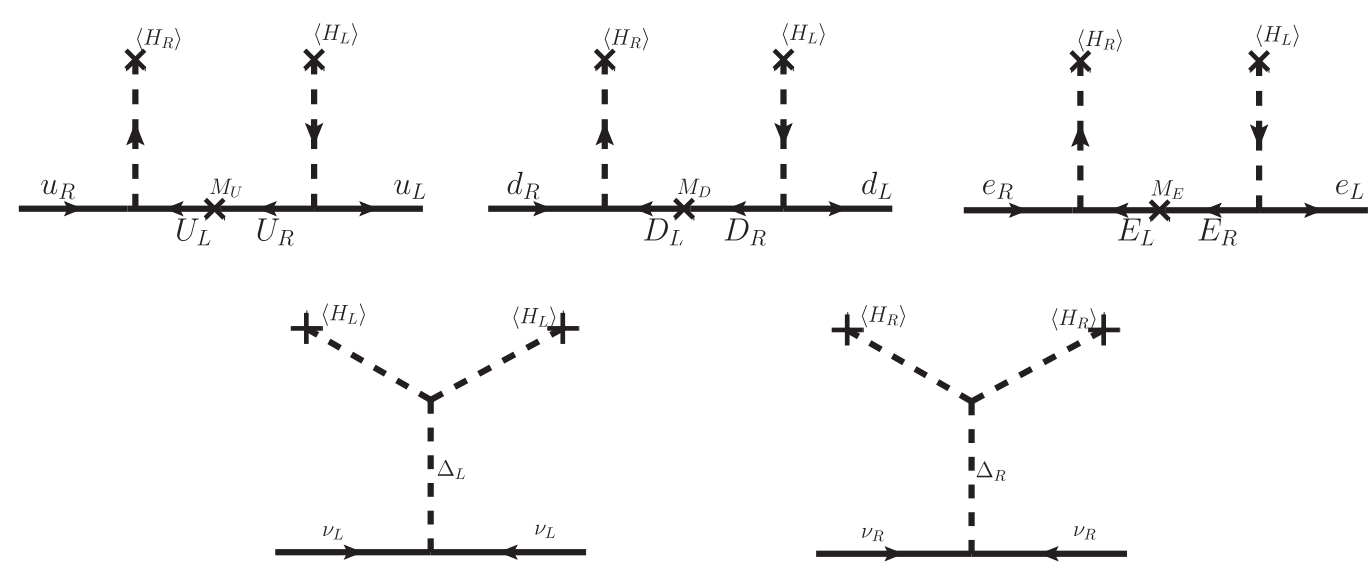

FIG. 1. Generation of fermion masses through universal seesaw and induced triplet VEVs.

Assuming all parameters to be real one can obtain the mass eigenstates by rotating the mass matrices via left and right orthogonal transformations $\mathcal{O}^{L, R}$. For example, up to leading order in $Y_{U}^{L} v_{L}$, the SM and heavy vector partner upquark masses are

$$
m_{u} \approx Y_{U}^{L} Y_{U}^{R} \frac{v_{L} v_{R}}{\hat{M}_{U}}, \quad \hat{M}_{U} \approx \sqrt{M_{U}^{2}+\left(Y_{U}^{R} v_{R}\right)^{2}},
$$

and the mixing angles $\theta_{U}^{L, R}$ in $\mathcal{O}^{L, R}$ are determined as

$$
\tan \left(2 \theta_{U}^{L, R}\right) \approx 2 Y_{U}^{L, R} \frac{v_{L, R} M_{U}}{M_{U}^{2} \pm\left(Y_{U}^{R} v_{R}\right)^{2}}
$$

The other fermion masses and mixing are obtained in an analogous manner. Note that here we have neglected the flavor structure of the Yukawa couplings $Y_{X}^{L, R}$ which will determine the observed quark and charged lepton mixings. The hierarchy of SM fermion masses can be explained by assuming either a hierarchical structure of the Yukawa couplings or a hierarchical structure of the vectorlike fermion masses.

\section{NEUTRINO MASSES AND TYPE II SEESAW DOMINANCE}

In the model under consideration there is no tree level Dirac mass term for the neutrinos due to the absence of a Higgs bidoublet. The scalar triplets acquire induced VEVs $\left\langle\Delta_{L}\right\rangle=u_{L}$ and $\left\langle\Delta_{R}\right\rangle=u_{R}$ giving the neutral lepton mass matrix in the basis $\left(\nu_{L}, \nu_{R}\right)$ given by

$$
M_{\nu}=\left(\begin{array}{cc}
f u_{L} & 0 \\
0 & f u_{R}
\end{array}\right) .
$$

Thus the light and heavy neutrino masses are simply $m_{\nu}=f u_{L} \propto M_{N}=f u_{R}$. A Dirac mass term is generated at the two-loop level via the one-loop $W$ boson mixing $\theta_{W}$ (see the next section) and the exchange of a charged lepton.
It is of the order $m_{D} \lesssim g_{L}^{4} /\left(16 \pi^{2}\right)^{2} m_{\tau} m_{b} m_{t} / M_{W_{R}}^{2} \approx 0.1 \mathrm{eV}$ for $M_{W_{R}} \approx 5 \mathrm{TeV}$. This is intriguingly of the order of the observed neutrino masses; as long as the right-handed neutrinos are much heavier than the left-handed neutrinos, the type-II seesaw dominance is preserved and the induced mixing $m_{D} / M_{N}$ is negligible. The mixing between charged gauge bosons $\theta_{W} \approx g_{L}^{2} /\left(16 \pi^{2}\right) m_{b} m_{t} / M_{W_{R}}^{2}$ is generated through the exchange of bottom and top quarks, and their vector-like partners. This yields a very small mixing of the order $\theta_{W} \approx 10^{-7}$ for TeV scale $W_{R}$ bosons.

Incorporating three fermion generations leads to the mixing matrices for the left- and right-handed matrices which we take to be equal

$$
V_{N}=V_{\nu} \equiv U
$$

where $U$ is the phenomenological Pontecorvo-MakiNakagawa-Sakata mixing matrix. Thus the unmeasured mixing matrix for the right-handed neutrinos is fully determined by the left-handed counterpart. The present framework gives a natural realization of type-II seesaw providing a direct relation between light and heavy neutrinos, $M_{i} \propto m_{i}$, i.e. the heavy neutrino masses $M_{i}$ can be expressed in terms of the light neutrino masses $m_{i}$ as $M_{i}=m_{i}\left(M_{3} / m_{3}\right)$, for a normal and $M_{i}=m_{i}\left(M_{2} / m_{2}\right)$ for a inverse hierarchy of light and heavy neutrino masses.

\section{GAUGE BOSONS}

As discussed in the previous section, we consider a scenario where the VEVs of the Higgs doublets are much larger than the VEVs of the Higgs triplets i.e, $u_{L} \ll v_{L}$, $u_{R} \ll v_{R}{ }^{2}$ Thus, the masses for the gauge bosons get small corrections from scalar triplets. The mass matrix for charged gauge bosons is given by

\footnotetext{
${ }^{2} \mathrm{An}$ interesting situation arises if one assumes $u_{L}$, $u_{R} \ll v_{L} \ll v_{R}$. Then one can allow right-handed Majorana neutrinos with masses below $\mathrm{GeV}$ which can play an important role in $0 \nu \beta \beta$ decay.
} 


$$
M_{W}^{2}=\frac{1}{4}\left(\begin{array}{cc}
g_{L}^{2}\left(v_{L}^{2}+2 u_{L}^{2}\right) & 0 \\
0 & g_{R}^{2}\left(v_{R}^{2}+2 u_{R}^{2}\right)
\end{array}\right),
$$

with the gauge couplings $g_{L}$ and $g_{R}$ associated with $S U(2)_{L}$ and $S U(2)_{R}$, respectively. The tree-level mixing between charged gauge bosons $W_{L}$ and $W_{R}$ is zero due to absence of a scalar bidoublet. The physical masses for charged gauge bosons are thus easily found,

$$
M_{W_{1}}^{2} \approx \frac{g_{L}^{2}}{4} v_{L}^{2}, \quad M_{W_{2}}^{2} \approx \frac{g_{R}^{2}}{4}\left(v_{R}^{2}+2 u_{R}^{2}\right),
$$

where we neglect $u_{L}$. At the one-loop level, a mixing of the order $\theta_{W} \approx g_{L}^{2} /\left(16 \pi^{2}\right) m_{b} m_{t} / M_{W_{R}}^{2}$ is generated through the exchange of bottom and top quarks, and their vectorlike partners. This yields a very small mixing of the order $\theta_{W} \approx$ $10^{-7}$ for $M_{W_{R}} \approx 5 \mathrm{TeV}$.

On the other hand, the neutral gauge boson mass matrix is given by

$$
M_{Z}^{2}=\frac{1}{4}\left(\begin{array}{ccc}
g_{L}^{2} v_{L}^{2} & 0 & -g_{L} g_{B L} \mu_{L}^{2} \\
0 & g_{R}^{2} \mu_{R}^{2} & -g_{B L} g_{R} \mu_{R}^{2} \\
-g_{B L} g_{L} \mu_{L}^{2} & -g_{B L} g_{R} \mu_{R}^{2} & g_{R}^{2} \mu_{L}^{2}+g_{B L}^{2} \mu_{R}^{2}
\end{array}\right),
$$

with $\mu_{L, R}^{2}=v_{L, R}^{2}+4 u_{L, R}^{2}$ and the gauge coupling $g_{B L}$ associated with $U(1)_{B-L}$. The diagonalization gives mass eigenvalues for the neutral gauge bosons,

$$
M_{Z_{1}}^{2} \approx \frac{g_{L}^{2}}{4 c_{W}^{2}} v_{L}^{2}, \quad M_{Z_{2}}^{2} \approx \frac{g_{B L}^{2}+g_{R}^{2}}{4}\left(v_{R}^{2}+4 u_{R}^{2}\right) .
$$

For $u_{L} \ll v_{L}, u_{R} \ll v_{R}$ the mixing between neutral gauge bosons $Z_{L}$ and $Z_{R}$ is given by $\theta_{Z} \approx v_{L}^{2} / v_{R}^{2} \simeq \mathcal{O}\left(10^{-4}\right)$. Thus, the mixing angle for neutral sector is much larger than the mixing angle between charged gauge boson. In fact, the absence of a sizeable mixing in the charged gauge boson and neutrino sector is a important consequence of our model and searches for $Z_{R}$ could constitute an important way of probing this scenario. Nevertheless, a mixing of order $\theta_{Z} \approx 10^{-4}$ is allowed by collider searches, electroweak precision data and searches for low-energy flavor changing neutral current processes [29-34], and we do not discuss potential avenues to probe the model in this direction but rather focus on neutrinoless double beta decay. We only comment that the mixing between $Z_{L}$ and $Z_{R}$ is a standard parameter being probed in collider experiments, for example at the LHC (see e.g. [35]) or especially at planned future electron-positron colliders. It could also be probed in upcoming searches for low-energy flavor changing neutral current processes.

\section{NEUTRINOLESS DOUBLE BETA DECAY}

As discussed earlier, there is no tree level Dirac neutrino mass term connecting light and heavy neutrinos. Consequently, the mixing between light and heavy neutrinos is vanishing at this order. Also, the mixing between the charged gauge bosons is vanishing at the tree level due to the absence of a scalar bidoublet.

The charged current interaction in the mass basis for the leptons is given by

$\frac{g_{L}}{\sqrt{2}} \sum_{i=1}^{3} U_{e i}\left(\bar{\ell}_{L} \gamma_{\mu} \nu_{i} W_{L}^{\mu}+\frac{g_{R}}{g_{L}} \bar{\ell}_{R} \gamma_{\mu} N_{i} W_{R}^{\mu}\right)+$ H.c.

The charged current interaction for leptons leads to $0 \nu \beta \beta$ decay via the exchange of light and heavy neutrinos. There are additional contributions to $0 \nu \beta \beta$ decay due to doubly charged triplet scalar exchange. While the left-handed triplet exchange is suppressed because of its small induced VEV, the right-handed triplet can contribute sizeably to $0 \nu \beta \beta$ decay.

Before numerical estimation, let us point out the mass relations between light and heavy neutrinos under natural type-II seesaw dominance. For a hierarchical pattern of light neutrinos the mass eigenvalues are given as $m_{1}<m_{2} \ll m_{3}$. The lightest neutrino mass eigenvalue is $m_{1}$ while the other mass eigenvalues are determined using the oscillation parameters as follows, $m_{2}^{2}=m_{1}^{2}+\Delta m_{\text {sol }}^{2}$, $m_{3}^{2}=m_{1}^{2}+\Delta m_{\mathrm{atm}}^{2}+\Delta m_{\mathrm{sol}}^{2}$. On the other hand, for the inverted hierarchical pattern of the light neutrino masses $m_{3} \ll m_{1} \approx m_{2}$ where $m_{3}$ is the lightest mass eigenvalue while other mass eigenvalues are determined by $m_{1}^{2}=m_{3}^{2}+\Delta m_{\mathrm{atm}}^{2}, m_{2}^{2}=m_{3}^{2}+\Delta m_{\mathrm{sol}}^{2}+\Delta m_{\mathrm{atm}}^{2}$. The quasidegenerate pattern of light neutrinos is $m_{1} \approx m_{2} \approx$ $m_{3} \gg \sqrt{\Delta m_{\mathrm{atm}}^{2}}$. In any case, the heavy neutrino masses are directly proportional to the light neutrino masses.

In the present analysis, we discuss $0 \nu \beta \beta$ decay due to exchange of light neutrinos via left-handed currents, righthanded neutrinos via right-handed currents as well as a right-handed doubly charged scalar $^{3}$ The half-life for a given isotope for these contributions is given by

$\left.\left[T_{1 / 2}^{0 \nu}\right]^{-1}=\left.G_{01}\left(\left|\mathcal{M}_{\nu} \eta_{\nu}\right|^{2}+\mid \mathcal{M}_{N}^{\prime} \eta_{N}+\mathcal{M}_{N} \eta_{\Delta}\right)\right|^{2}\right)$

where $G_{01}$ corresponds to the standard $0 \nu \beta \beta$ phase space factor, the $\mathcal{M}_{i}$ correspond to the nuclear matrix elements for the different exchange processes and $\eta_{i}$ are dimensionless parameters determined below.

\footnotetext{
${ }^{3}$ A detailed discussion of $0 \nu \beta \beta$ decay within LRSMs can be found e.g. in Refs. [9,28,36-44] and for an early study of the effects of light and heavy Majorana neutrinos in neutrinoless double beta decay see Ref. [45].
} 


\section{A. Light neutrinos}

The lepton number violating dimensionless particle physics parameter derived from $0 \nu \beta \beta$ decay due to the standard mechanism via the exchange of light neutrinos is

$$
\eta_{\nu}=\frac{1}{m_{e}} \sum_{i=1}^{3} U_{e i}^{2} m_{i}=\frac{m_{\mathrm{ee}}^{\nu}}{m_{e}} .
$$

Here, $m_{e}$ is the electron mass and the effective $0 \nu \beta \beta$ mass is explicitly given by

$$
m_{\mathrm{ee}}^{\nu}=\left|c_{12}^{2} c_{13}^{2} m_{1}+s_{12}^{2} c_{13}^{2} m_{2} e^{i \alpha}+s_{13}^{2} m_{3} e^{i \beta}\right|,
$$

with the sine and cosine of the oscillation angles $\theta_{12}$ and $\theta_{13}, c_{12}=\cos \theta_{12}$, etc. and the unconstrained Majorana phases $0 \leq \alpha, \beta<2 \pi$.

\section{B. Right-handed neutrinos}

The contribution to $0 \nu \beta \beta$ decay arising from the purely right-handed currents via the exchange of right-handed neutrinos generally results in the lepton number violating dimensionless particle physics parameter

$$
\eta_{N}=m_{p}\left(\frac{g_{R}}{g_{L}}\right)^{4}\left(\frac{M_{W_{L}}}{M_{W_{R}}}\right)^{4} \sum_{i=1}^{3} \frac{U_{e i}^{2} M_{i}}{|p|^{2}+M_{i}^{2}} .
$$

The virtual neutrino momentum $|p|$ is of the order of the nuclear Fermi scale, $p \approx 100 \mathrm{MeV} . m_{p}$ is the proton mass and for the manifest LRSM case we have $g_{L}=g_{R}$, or else the new contributions are rescaled by the ratio between these two couplings. We in general consider right-handed neutrinos that can be either heavy or light compared to nuclear Fermi scale.

If the mass of the exchanged neutrino is much higher than its momentum, $M_{i} \gg|p|$, the propagator simplifies as

$$
\frac{M_{i}}{p^{2}-M_{i}^{2}} \approx-\frac{1}{M_{i}},
$$

and the effective parameter for right-handed neutrino exchange yields

$$
\eta_{N}=m_{p}\left(\frac{g_{R}}{g_{L}}\right)^{4}\left(\frac{M_{W}}{M_{W_{R}}}\right)^{4} \sum_{i=1}^{3} \frac{U_{e i}^{2}}{M_{i}} \propto \eta_{\nu}\left(m_{i}^{-1}\right),
$$

where in the expression for $\eta_{\nu}\left(m_{i}^{-1}\right)$ the individual neutrino masses are replaced by their inverse values. Such a contribution clearly becomes suppressed the smaller the right-handed neutrino masses are.

On the other hand, if the mass of the neutrino is much less than its typical momentum, $M_{i} \ll|p|$, the propagator simplifies in the same way as for the light neutrino exchange,

$$
P_{R} \frac{\not p+M_{i}}{p^{2}-M_{i}^{2}} P_{R} \approx \frac{M_{i}}{p^{2}}
$$

because both currents are right-handed. As a result, the $0 \nu \beta \beta$ decay contribution leads to the dimensionless parameter

$$
\eta_{N}=\frac{m_{p}}{|p|^{2}}\left(\frac{g_{R}}{g_{L}}\right)^{4}\left(\frac{M_{W}}{M_{W_{R}}}\right)^{4} \sum_{i=1}^{3} U_{e i}^{2} M_{i} \propto \eta_{\nu} .
$$

This is proportional to the standard parameter $\eta_{\nu}$ but in the case of very light right-handed neutrinos, e.g. $M_{i} \approx m_{i}$, the contribution becomes negligible because of the strong suppression with the heavy right-handed $W$ boson mass.

In general, we consider right-handed neutrinos both lighter and heavier than $100 \mathrm{MeV}$ and use (26) to calculate the contribution. In addition, the relevant nuclear matrix element changes; for $M_{i} \gg 100 \mathrm{MeV}$ it approaches $\mathcal{M}_{N}^{\prime} \rightarrow \mathcal{M}_{N}$ whereas for $M_{i} \ll 100 \mathrm{MeV}$ it approaches $\mathcal{M}_{N}^{\prime} \rightarrow \mathcal{M}_{\nu}$. For intermediate values, we use a simple smooth interpolation scheme within the regime $10 \mathrm{MeV}-$ $1 \mathrm{GeV}$, which yields a sufficient accuracy for our purposes.

\section{Right-handed triplet scalar}

Finally, the exchange of a doubly charged right-handed triplet scalar gives

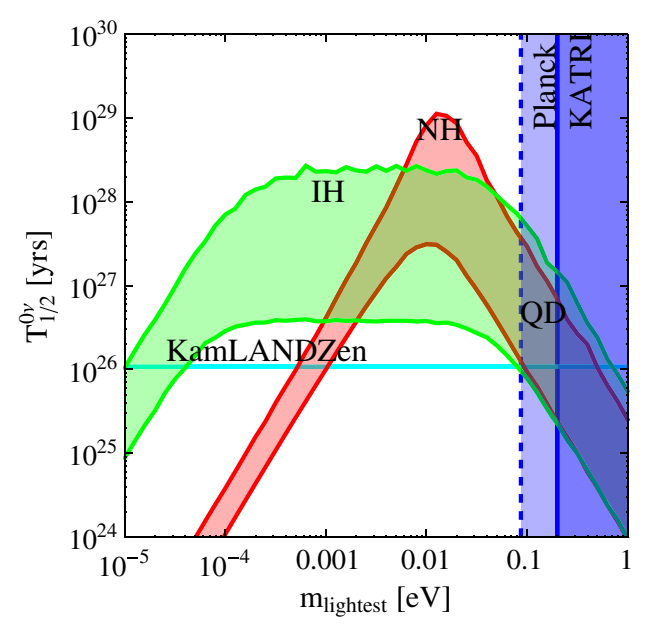

FIG. 2. $0 \nu \beta \beta$ decay half-life as a function of the lightest neutrino mass in the case of normal hierarchical $(\mathrm{NH})$ and inverse hierarchical (IH) light neutrinos in red and green bands respectively. The other parameters are fixed as $M_{W_{R}}=5 \mathrm{TeV}$, $M_{\delta_{R}^{--}} \approx 5 \mathrm{TeV}$ and the heaviest right-handed neutrino mass is $1 \mathrm{TeV}$. The gauge couplings are assumed universal, $g_{L}=g_{R}$, and the intermediate values for the nuclear matrix elements are used, $\mathcal{M}_{\nu}=4.5, \mathcal{M}_{N}=270$. The bound on the sum of light neutrino masses from the KATRIN and Planck experiments are represented as vertical lines. The bound from KamLAND-Zen experiment is presented in horizontal line for Xenon isotope. The bands arise due to $3 \sigma$ range of neutrino oscillation parameters and variation in the Majorana phases from $0-2 \pi$. 
TABLE II. Phase space factor $G_{01}$ and ranges of nuclear matrix elements for light and heavy neutrino exchange for the isotopes ${ }^{76} \mathrm{Ge}$ and ${ }^{136} \mathrm{Xe}$ [47].

\begin{tabular}{lccc}
\hline \hline Isotope & $G_{01}\left(\mathrm{yr}^{-1}\right)$ & $\mathcal{M}_{\nu}$ & $\mathcal{M}_{N}$ \\
\hline${ }^{76} \mathrm{Ge}$ & $5.77 \times 10^{-15}$ & $2.58-6.64$ & $233-412$ \\
${ }^{136} \mathrm{Xe}$ & $3.56 \times 10^{-14}$ & $1.57-3.85$ & $164-172$ \\
\hline \hline
\end{tabular}

$$
\eta_{\Delta}=\frac{m_{p}}{M_{\delta_{R}^{--}}^{2}}\left(\frac{g_{R}}{g_{L}}\right)^{4}\left(\frac{M_{W}}{M_{W_{R}}}\right)^{4} \sum_{i=1}^{3} U_{e i}^{2} M_{i} \propto \eta_{\nu} .
$$

This expression is also proportional to the standard $\eta_{\nu}$ because the relevant coupling of the triplet scalar is proportional to the right-handed neutrino mass.

\section{Numerical estimate}

In the following, we numerically estimate the half-life for $0 \nu \beta \beta$ decay of the isotope ${ }^{136} \mathrm{Xe}$ as shown in Fig. 2. We use the current values of masses and mixing parameters from neutrino oscillation data reported in the global fits taken from Ref. [46]. For the $0 \nu \beta \beta$ phase space factors and nuclear matrix elements we use the values given in Table II. In Fig. 2, we show the dependence of the $0 \nu \beta \beta$ decay halflife on the lightest neutrino mass, i.e. $m_{1}$ for normal and $m_{3}$ for inverse hierarchical neutrinos. The other model parameters are fixed as

$g_{R}=g_{L}, \quad M_{W_{R}}=M_{\delta_{R}^{--}} \approx 5 \mathrm{TeV}, \quad M_{N}^{\text {heaviest }}=1 \mathrm{TeV}$.

The lower limit on lightest neutrino mass is derived to be $m_{<} \approx 0.9 \mathrm{meV}, 0.01 \mathrm{meV}$ for $\mathrm{NH}$ and $\mathrm{IH}$ pattern of light neutrino masses respectively by saturating the KamLAND-Zen experimental bound.

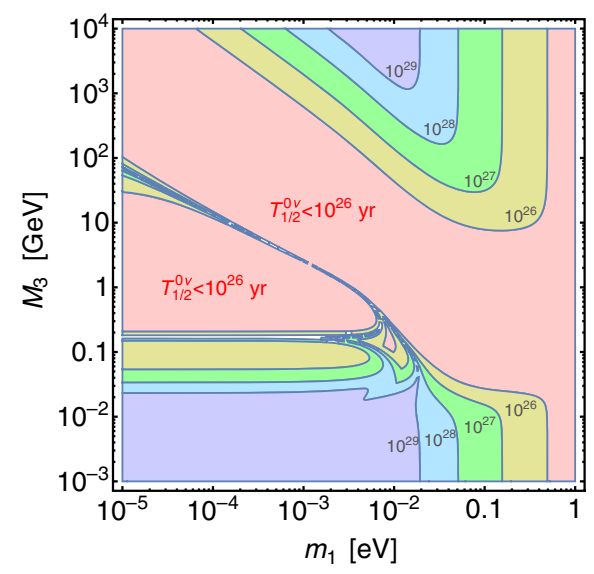

As for the experimental constraints, we use the current best limits at $90 \%$ C.L., $T_{1 / 2}^{0 \nu}\left({ }^{136} \mathrm{Xe}\right)>1.07 \times 10^{26} \mathrm{yr}$ and $T_{1 / 2}^{0 \nu}\left({ }^{76} \mathrm{Ge}\right)>2.1 \times 10^{25} \mathrm{yr}$ from KamLAND-Zen [3] and the GERDA Phase I [48], respectively. Representative for the sensitivity of future $0 \nu \beta \beta$ experiments, we use the expected reach of the planned $\mathrm{nEXO}$ experiment, $T_{1 / 2}^{0 \nu}\left({ }^{136} \mathrm{Xe}\right) \approx 6.6 \times 10^{27} \mathrm{yr}[49]$. As for the other experimental probes on the neutrino mass scale, we use the future sensitivity of the KATRIN experiment on the effective single $\beta$ decay mass $m_{\beta} \approx 0.2 \mathrm{eV} \mathrm{[50]} \mathrm{and} \mathrm{the} \mathrm{current} \mathrm{limit}$ on the sum of neutrino masses from cosmological observations, $\Sigma_{i} m_{i} \lesssim 0.7 \mathrm{eV}[51]$.

For a better understand of the interplay between the leftand right-handed neutrino mass scales, we show in Fig. 3 the $0 \nu \beta \beta$ decay half-life as a function of the lightest neutrino mass and the heaviest neutrino mass for a normal (left) and inverse (right) neutrino mass hierarchy. The other model parameters are fixed, with right-handed gauge boson and doubly-charged scalar masses of $5 \mathrm{TeV}$. The oscillation parameters are at their best fit values and the Majorana phases are always chosen to yield the smallest rate at a given point, i.e. the longest half life. The nuclear matrix employed are at the lower end in Table II. This altogether yields the longest, i.e. most pessimistic, prediction for the $0 \nu \beta \beta$ decay half-life. The red-shaded area is already excluded with a predicted half life of $10^{26} \mathrm{yr}$ or faster. As expected, this sets an upper limit on the lightest neutrino mass $m_{\text {lightest }} \lesssim 1 \mathrm{eV}$, but it also puts stringent constraints on the mass scale of the right-handed neutrinos. For an inverse hierarchy, the range $50 \mathrm{MeV} \lesssim M_{2} \lesssim 5 \mathrm{GeV}$ is excluded whereas in the normal hierarchy case, large $M_{3}$ can be excluded if there is a strong hierarchy, $m_{1} \rightarrow 0$. This is due the large contribution of the lightest heavy neutrino $N_{1}$ in such a case.

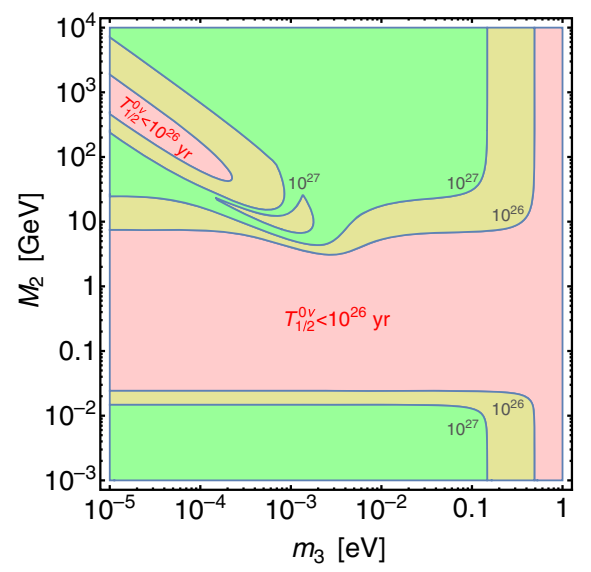

FIG. 3. Half-life of $0 \nu \beta \beta$ decay in Xe as a function of the lightest and the heaviest neutrino mass for a normal (left) and inverse (right) neutrino mass hierarchy. The contours denote the half-life in years. Best-fit oscillation data are used and the Majorana phases are chosen to yield the longest half-life. Likewise, the smallest values of the nuclear matrix elements in Table II are employed. The other model parameters are chosen as $g_{R}=g_{L}$ and $M_{W_{R}}=M_{\Delta}=5 \mathrm{TeV}$. 


\section{CONCLUSION}

We have presented a left-right symmetric model with additional vectorlike fermions in order to simultaneously explain the charged fermion and Majorana neutrino masses. The quark and charged lepton masses and mixing is realized via a universal seesaw mechanism. Although spontaneous symmetry breaking is achieved with two doublet Higgs fields with nonzero $B-L$ charge, we have introduced scalar triplets with small induced VEVs such that they give Majorana masses to light as well as heavy neutrinos. The Majorana nature of these neutrinos leads to $0 \nu \beta \beta$ decay and it is found that the right-handed currents play an important role in discriminating between the mass hierarchy as well as the absolute scale of light neutrinos.

\section{ACKNOWLEDGMENTS}

P. P. is supported by the DST INSPIRE Fellowship (No. DST/INSPIRE Fellowship/2014/IF140299) funded by the Department of Science and Technology, India. The work of S.P. is partially supported by the Department of Science and Technology, Govt. of India under the financial Grant No. SB/S2/HEP-011/2013. The work of U.S. is supported partly by the JC Bose National Fellowship grant under DST, India.
[1] P. A. M. Dirac, The fundamental equations of quantum mechanics, Proc. R. Soc. London 109, 642 (1925).

[2] E. Majorana, Teoria simmetrica dell'elettrone e del positrone, Nuovo Cimento 14, 171 (1937).

[3] A. Gando et al. (KamLAND-Zen Collaboration), Search for Majorana Neutrinos Near the Inverted Mass Hierarchy Region with KamLAND-Zen, Phys. Rev. Lett. 117, 082503 (2016); Publisher's Note, Phys. Rev. Lett. 117, 109903 (2016).

[4] J. C. Pati and A. Salam, Lepton number as the fourth color, Phys. Rev. D 10, 275 (1974).

[5] R. Mohapatra and J. C. Pati, A natural left-right symmetry, Phys. Rev. D 11, 2558 (1975).

[6] G. Senjanović and R. N. Mohapatra, Exact left-right symmetry and spontaneous violation of parity, Phys. Rev. D 12, 1502 (1975).

[7] G. Senjanović, Spontaneous breakdown of parity in a class of gauge theories, Nucl. Phys. B153, 334 (1979).

[8] R. N. Mohapatra and G. Senjanovic, Neutrino Mass and Spontaneous Parity Violation, Phys. Rev. Lett. 44, 912 (1980).

[9] R. N. Mohapatra and G. Senjanović, Neutrino masses and mixings in gauge models with spontaneous parity violation, Phys. Rev. D 23, 165 (1981).

[10] P. Minkowski, $\mu \rightarrow e \gamma$ at a rate of one out of $10^{9}$ muon decays?, Phys. Lett. 67B, 421 (1977).

[11] M. Gell-Mann, P. Ramond, and R. Slansky, Complex spinors and unified theories, Conference Proceedings C790927, 315 (1979).

[12] T. Yanagida, Horizontal symmetry and masses of neutrinos, Conference Proceedings C7902131, 95 (1979).

[13] J. Schechter and J. W. F. Valle, Neutrino masses in $\mathrm{SU}(2) \times$ U(1) theories, Phys. Rev. D 22, 2227 (1980).

[14] J. Schechter and J. W. F. Valle, Neutrino decay and spontaneous violation of lepton number, Phys. Rev. D 25, 774 (1982).

[15] V. Khachatryan et al. (CMS Collaboration), Search for heavy neutrinos and $\mathrm{W}$ bosons with right-handed couplings in proton-proton collisions at $\sqrt{s}=8 \mathrm{TeV}$, Eur. Phys. J. C 74, 3149 (2014).
[16] G. Beall, M. Bander, and A. Soni, Constraint on the Mass Scale of a Left-Right Symmetric Electroweak Theory from the $\mathrm{K}(\mathrm{L}) \mathrm{K}(\mathrm{S})$ Mass Difference, Phys. Rev. Lett. 48, 848 (1982).

[17] Y. Zhang, H. An, X. Ji, and R. N. Mohapatra, General CP violation in minimal left-right symmetric model and constraints on the right-handed scale, Nucl. Phys. B802, 247 (2008).

[18] A. Maiezza, M. Nemevsek, F. Nesti, and G. Senjanovic, Left-right symmetry at LHC, Phys. Rev. D 82, 055022 (2010).

[19] S. Bertolini, A. Maiezza, and F. Nesti, Present and future K and $\mathrm{B}$ meson mixing constraints on $\mathrm{TeV}$ scale left-right symmetry, Phys. Rev. D 89, 095028 (2014).

[20] F. F. Deppisch, T. E. Gonzalo, S. Patra, N. Sahu, and U. Sarkar, Signal of right-handed charged gauge bosons at the LHC?, Phys. Rev. D 90, 053014 (2014).

[21] F. F. Deppisch, T. E. Gonzalo, S. Patra, N. Sahu, and U. Sarkar, Double beta decay, lepton flavor violation, and collider signatures of left-right symmetric models with spontaneous D-parity breaking, Phys. Rev. D 91, 015018 (2015).

[22] F. F. Deppisch, L. Graf, S. Kulkarni, S. Patra, W. Rodejohann, N. Sahu, and U. Sarkar, Reconciling the $2 \mathrm{TeV}$ excesses at the LHC in a linear seesaw left-right model, Phys. Rev. D 93, 013011 (2016).

[23] M. Lindner, F. S. Queiroz, and W. Rodejohann, Dilepton bounds on left-right symmetry at the LHC run II and neutrinoless double beta decay, Phys. Lett. B 762, 190 (2016).

[24] S. Patra, F. S. Queiroz, and W. Rodejohann, Stringent dilepton bounds on left-right models using LHC data, Phys. Lett. B 752, 186 (2016).

[25] P. S. B. Dev, R. N. Mohapatra, and Y. Zhang, Quark seesaw, vectorlike fermions and diphoton excess, J. High Energy Phys. 02 (2016) 186.

[26] F. F. Deppisch, C. Hati, S. Patra, P. Pritimita, and U. Sarkar, Implications of the diphoton excess on left-right models and gauge unification, Phys. Lett. B 757, 223 (2016). 
[27] E. Gabrielli, L. Marzola, and M. Raidal, Radiative Yukawa couplings in the simplest left-right symmetric model, Phys. Rev. D 95, 035005 (2017).

[28] S. Patra, Neutrinoless double beta decay process in left-right symmetric models without scalar bidoublet, Phys. Rev. D 87, 015002 (2013).

[29] J. Polak and M. Zralek, Updated constraints on the neutral current parameters in left-right symmetric model, Nucl. Phys. B363, 385 (1991).

[30] J. Beringer et al. (Particle Data Group), Review of particle physics, Phys. Rev. D 86, 010001 (2012).

[31] J. Erler, P. Langacker, S. Munir, and E. Rojas, Improved constraints on Z-prime bosons from electroweak precision data, J. High Energy Phys. 08 (2009) 017.

[32] T. Aaltonen et al. (CDF Collaboration), Search for $W W$ and $W Z$ Resonances Decaying to Electron, Missing $E_{T}$, and Two Jets in $p \bar{p}$ Collisions at $\sqrt{s}=1.96 \mathrm{TeV}$, Phys. Rev. Lett. 104, 241801 (2010).

[33] V. V. Andreev and A. A. Pankov, Constraints on the Z-Zprime mixing angle from data measured for the process $\mathrm{e}+\mathrm{e}-$ to $\mathrm{W}+\mathrm{W}-$ at the LEP2 collider, Phys. At. Nucl. 75, 76 (2012).

[34] P. Osland, A. A. Pankov, and A. V. Tsytrinov, Probing $Z-Z^{\prime}$ mixing with ATLAS and CMS resonant diboson production data at the LHC at $\sqrt{s}=13 \mathrm{TeV}$, Phys. Rev. D 96, 055040 (2017).

[35] F. F. Deppisch, N. Desai, and J. W. F. Valle, Is charged lepton flavor violation a high energy phenomenon?, Phys. Rev. D 89, 051302 (2014).

[36] R. N. Mohapatra and J. D. Vergados, A New Contribution to Neutrinoless Double Beta Decay in Gauge Models, Phys. Rev. Lett. 47, 1713 (1981).

[37] M. Hirsch, H. V. Klapdor-Kleingrothaus, and O. Panella, Double beta decay in left-right symmetric models, Phys. Lett. B 374, 7 (1996).

[38] V. Tello, M. Nemevsek, F. Nesti, G. Senjanovic, and F. Vissani, Left-Right Symmetry: from LHC to Neutrinoless Double Beta Decay, Phys. Rev. Lett. 106, 151801 (2011).

[39] J. Chakrabortty, H. Z. Devi, S. Goswami, and S. Patra, Neutrinoless double- $\beta$ decay in TeV scale left-right symmetric models, J. High Energy Phys. 08 (2012) 008.
[40] R. L. Awasthi, M. Parida, and S. Patra, Neutrino masses, dominant neutrinoless double beta decay, and observable lepton flavor violation in left-right models and $\mathrm{SO}(10)$ grand unification with low mass $W_{R}, Z_{R}$ bosons, J. High Energy Phys. 08 (2013) 122.

[41] J. Barry and W. Rodejohann, Lepton number and flavour violation in $\mathrm{TeV}$-scale left-right symmetric theories with large left-right mixing, J. High Energy Phys. 09 (2013) 153.

[42] P. Bhupal Dev, S. Goswami, M. Mitra, and W. Rodejohann, Constraining neutrino mass from neutrinoless double beta decay, Phys. Rev. D 88, 091301 (2013).

[43] S.-F. Ge, M. Lindner, and S. Patra, New physics effects on neutrinoless double beta decay from right-handed current, J. High Energy Phys. 10 (2015) 077.

[44] R. L. Awasthi, P. S. B. Dev, and M. Mitra, Implications of the diboson excess for neutrinoless double beta decay and lepton flavor violation in $\mathrm{TeV}$ scale left right symmetric model, Phys. Rev. D 93, 011701 (2016).

[45] A. Halprin, S. T. Petcov, and S. P. Rosen, Effects of light and heavy Majorana neutrinos in neutrinoless double beta decay, Phys. Lett. 125B, 335 (1983).

[46] M. C. Gonzalez-Garcia, M. Maltoni, J. Salvado, and T. Schwetz, Global fit to three neutrino mixing: critical look at present precision, J. High Energy Phys. 12 (2012) 123.

[47] A. Meroni, S. T. Petcov, and F. Simkovic, Multiple $C P$ nonconserving mechanisms of $(\beta \beta)_{0 \nu}$-decay and nuclei with largely different nuclear matrix elements, J. High Energy Phys. 02 (2013) 025.

[48] M. Agostini et al. (GERDA Collaboration), Results on Neutrinoless Double- $\beta$ Decay of ${ }^{76} \mathrm{Ge}$ from Phase I of the GERDA Experiment, Phys. Rev. Lett. 111, 122503 (2013).

[49] J. Albert, Status and results from the EXO Collaboration, EPJ Web Conf. 66, 08001 (2014).

[50] S. Mertens (KATRIN Collaboration), Status of the KATRIN Experiment and prospects to search for keV-mass sterile neutrinos in tritium $\beta$-decay, Phys. Procedia 61, 267 (2015).

[51] P. A. R. Ade et al. (Planck Collaboration), Planck 2015 results. XIII. Cosmological parameters, Astron. Astrophys. 594, A13 (2016). 